\title{
ON THE EXACT CONTROLLABILITY OF A NONLINEAR STOCHASTIC HEAT EQUATION
}

\author{
BUI AN TON
}

Received 12 December 2004; Accepted 20 January 2005

The exact controllability of a nonlinear stochastic heat equation with null Dirichlet boundary conditions, nonzero initial and target values, and an interior control is established.

Copyright (c) 2006 Bui An Ton. This is an open access article distributed under the Creative Commons Attribution License, which permits unrestricted use, distribution, and reproduction in any medium, provided the original work is properly cited.

\section{Introduction}

The purpose of this paper is to establish the exact controllability of a nonlinear stochastic heat equation with null Dirichlet boundary conditions, nonzero initial and target values.

Let $(\Omega, \mathcal{A}, P)$ be a probability space, let $\mathscr{F}^{t}$ be a filtration on the space and let $w$ be a $\mathscr{F}^{t}$ standard Wiener process. Given two elements $\alpha, \beta$ of $L^{2}(G)$, one wishes to find a control $v$ and $y$ such that

$$
\begin{gathered}
d y-\Delta y d t+f d w=v d t+g(y) d t \quad \text { in } G \times(0, T), \\
y(x, t ; \omega)=0 \quad \text { on } \partial G \times(0, T), \text { a.s. } \\
y(x, 0 ; \omega)=\alpha ; \quad y(x, T ; \omega)=\beta \quad \text { in } G \text { a.s. }
\end{gathered}
$$

The bounded open subset $G$ of $R^{n}$ is assumed to have a smooth boundary and $g$ is continuous from $L^{2}\left(0, T ; L^{2}(G)\right)$ into $L^{2}\left(0, T ; L^{2}(G)\right)$. The existence of a solution of the linear version of (1.1) is established in Section 3 of the paper. The nonlinear case is considered in Section 4.

The main assumptions of the paper and some preliminary results are given in Section 2.

Exact controllability of the wave equation has been the subject of extensive investigations using the HUM method of J. L. Lions and the nonlinear case has been studied by Zuazua [6] and recently by the author in [1]. 
In contrast with the hyperbolic case, there are few works on the exact controllability of parabolic equations and studies for the nonlinear stochastic heat equation seem non existent. For the linear deterministic heat equation, the exact controllability was established by Lebeau and Robbiano [4] and earlier by Russell [5] with some additional hypotheses. For the deterministic semilinear heat equation, the approximate exact controllability was shown by Fabre et al. [3]. The result of the paper on the exact controllability for the nonlinear stochastic heat equation seems new.

\section{Some preliminary results}

In this section we will consider the exact controllability of the linear heat equation. Consider the problem

$$
\begin{gathered}
z^{\prime}-\Delta z=v \quad \text { in } G \times(0, T), \text { a.s., } \\
z(x, t ; \omega)=0 \quad \text { on } \partial G \times(0, T), \text { a.s., } \\
z(x, 0 ; \omega)=\alpha ; \quad z(x, T ; \omega)=\gamma(x ; \omega) \quad \text { in } G \text { a.s. }
\end{gathered}
$$

Theorem 2.1. Let $\{\alpha, \gamma\}$ be in $L^{2}(G) \times L^{2}\left(\Omega, \mathcal{A}, P ; L^{2}(G)\right)$, then there exists a solution $\{v, z\}$ of (2.1) in

$$
L^{2}\left(\Omega, \mathscr{A} ; P ; L^{2}\left(0, T ; H^{-1}(G)\right)\right) \times L^{2}\left(\Omega, \mathscr{A}, P ; C\left(0, T ; L^{2}(G)\right) \cap L^{2}\left(0, T ; H_{0}^{1}(G)\right)\right) .
$$

Moreover

$$
\begin{aligned}
& E\left(\|z\|_{L^{2}\left(0, T ; H_{0}^{1}(G)\right)}^{2}\right)+E\left(\|z\|_{L^{\infty}\left(0, T ; L^{2}(G)\right)}^{2}\right)+E\left(\left\|z^{\prime}\right\|_{L^{2}\left(0, T ; H^{-1}(G)\right)}^{2}\right)+E\left(\|v\|_{L^{2}\left(0, T ; H^{-1}(G)\right)}^{2}\right) \\
& \quad \leq C\left\{\|\alpha\|_{L^{2}(G)}^{2}+E\left(\|\gamma\|_{L^{2}(G)}^{2}\right)\right\} .
\end{aligned}
$$

The constant $C$ is independent of $\alpha, \gamma$.

Consider the initial boundary-value problem

$$
\begin{gathered}
\hat{z}^{\prime}-\Delta \hat{z}=0 \quad \text { in } G \times(0, T), \\
\hat{z}(x, t)=0 \quad \text { on } \partial G \times(0, T), \\
z(x, 0)=\alpha \quad \text { in } G .
\end{gathered}
$$

Lemma 2.2. There exists a unique solution $\hat{z}$ of (2.4) with

$$
\|\hat{z}\|_{L^{2}\left(0, T ; H_{0}^{1}(G)\right)}+\left\|\hat{z}^{\prime}\right\|_{L^{2}\left(0, T ; H^{-1}(G)\right)} \leq C\|\alpha\|_{L^{2}(G)} .
$$

The constant $C$ is independent of $\alpha$. 
We now consider the exact controllability of the problem

$$
\begin{gathered}
\tilde{z}^{\prime}-\Delta \tilde{z}=\tilde{v} \quad \text { in } G \times(0, T), \\
\tilde{z}(x, t)=0 \quad \text { on } \partial G \times(0, T), \\
\tilde{z}(x, 0)=\gamma-\hat{z}(x, T) ; \quad \tilde{z}(x, T)=0 \quad \text { in } G .
\end{gathered}
$$

First we consider the deterministic case.

Lemma 2.3. Let $\alpha, \gamma$ be in $L^{2}(G)$ and let $\hat{z}$ be as in Lemma 2.2. Then there exists a solution $\{\tilde{v}, \tilde{z}\}$ of $(2.6)$ in

$$
L^{\infty}\left(0, T ; L^{2}(G)\right) \times C\left(0, T ; L^{2}(G)\right) \cap L^{2}\left(0, T ; H_{0}^{1}(G)\right) .
$$

Moreover

$$
\|\tilde{z}\|_{L^{2}\left(0, T ; H_{0}^{1}(G)\right)}+\left\|\tilde{z}^{\prime}\right\|_{L^{2}\left(0, T ; H^{-1}(G)\right)}+\|\tilde{v}\|_{L^{\infty}\left(0, T ; L^{2}(G)\right)} \leq C\left\{\|\alpha\|_{L^{2}(G)}+\|\gamma\|_{L^{2}(G)}\right\} .
$$

The constant $C$ is independent of $\alpha, \gamma, \hat{z}$.

Proof. It follows from [4, Theorem 1 (page 336) and from (35)-(37) (page 344)] that there exists $\{\tilde{v}, \tilde{z}\} \in L^{\infty}\left(0, T ; L^{2}(G)\right) \times L^{2}\left(0, T ; H_{0}^{1}(G)\right)$, solution of (2.6). Moreover

$$
\|\tilde{v}\|_{L^{\infty}\left(0, T ; L^{2}(G)\right)} \leq C\|\gamma-\hat{z}(\cdot, T)\|_{L^{2}(G)} \leq C\left\{\|\alpha\|_{L^{2}(G)}+\|\gamma\|_{L^{2}(G)}\right\} .
$$

With the above estimate for $\tilde{v}$, the stated result is now obvious

Let $s=T-t, \widetilde{z}(x, t)=\widetilde{z}(x, T-s)=\bar{z}(x, s)$, then we have

$$
\begin{gathered}
\bar{z}^{\prime}+\Delta \bar{z}=-\widetilde{v} \quad \text { in } G \times(0, T), \\
\bar{z}(x, s)=0 \quad \text { on } \partial G \times(0, T), \\
\bar{z}(x, 0)=0 ; \quad \bar{z}(x, T)=\gamma-\widehat{z}(x, T) \quad \text { in } G .
\end{gathered}
$$

Set $z_{*}=\bar{z}+\hat{z}$, then we get

$$
\begin{gathered}
z_{*}^{\prime}-\Delta z_{*}=-\tilde{v}-2 \Delta \bar{z}=\hat{v} \quad \text { in } G \times(0, T), \\
z_{*}(x, t)=0 \quad \text { on } \partial G \times(0, T), \\
z_{*}(x, 0)=\alpha ; \quad z_{*}(x, T)=\gamma \quad \text { in } G .
\end{gathered}
$$

Let $\Gamma$ be the set-valued mapping

$$
\Gamma(\gamma)=\left\{\left[\hat{v}, z_{*}\right]:\left\{\widehat{v}, z_{*}\right\} \text { solution of }(2.11)\right\}
$$

of $L^{2}(G)$ into $L^{2}\left(0, T ; H^{-1}(G)\right) \times\left\{L^{2}\left(0, T ; H_{0}^{1}(G)\right) \cap C\left(0, T ; L^{2}(G)\right)\right.$.

Lemma 2.4. Let $\Gamma$ be as in (2.12), then the images of $\Gamma$ are closed subsets of $L^{2}\left(0, T ; H^{-1}(G)\right)$ $\times\left\{L^{2}\left(0, T ; H_{0}^{1}(G)\right) \cap C\left(0, T ; L^{2}(G)\right)\right\}$. Moreover the graph of $\Gamma$ is closed. 
Proof. Since the problem is linear, in view of the estimates of Lemmas 2.2, 2.3 it is clear that the graph of $\Gamma$ is closed and that its images are nonempty closed subsets of

$$
L^{2}\left(0, T ; H^{-1}(G)\right) \times L^{2}\left(0, T ; H_{0}^{1}(G)\right) \cap C\left(0, T ; L^{2}(G)\right) .
$$

We now consider (2.11) when $\gamma$ is in $L^{2}\left(\Omega, \mathscr{A}, P ; L^{2}(G)\right)$.

Let $\varphi$ be the random variable

$$
\varphi(\omega)=\gamma(x ; \omega)
$$

of $\Omega$ into $L^{2}(G)$ and set

$$
\Lambda(\omega)=\Gamma \circ \varphi(\omega)=\left\{\left[\hat{v}(\cdot, \omega), z_{*}(\cdot, \omega)\right] \text {; solution of }(2.11)\right\}
$$

Lemma 2.5. The set valued mapping $\Gamma$ given by (2.11) has a universally measurable section $\sigma$. The application $\sigma \circ \varphi$ of

$$
\Omega \longrightarrow L^{2}\left(0, T ; H^{-1}(G)\right) \times L^{2}\left(0, T ; H_{0}^{1}(G)\right)
$$

is a measurable section of $\Lambda$.

Proof. (1) Since the set valued mapping $\Gamma$ has nonempty closed images in $L^{2}(0, T$; $\left.H^{-1}(G)\right) \times L^{2}\left(0, T ; H_{0}^{1}(G)\right) \cap C\left(0, T ; L^{2}(G)\right)$ with closed graph, it follows from a theorem of Von Neumann that there exists a universally measurable section $\sigma$ of $\Gamma$. (cf. [2, Theorem 3.1, page 206]).

(2) Since $P$ is a Radon measure on a regular space and since $\varphi$ is a random variable and hence is measurable, for each $k$ there exists a compact $K_{k}$ of $\Omega$ such that

$$
P\left(\Omega / K_{k}\right) \leq 1 / k
$$

We may assume without loss of generality that $K_{k}$ is an increasing sequence.

The restriction $\varphi_{k}=\left.\varphi\right|_{K_{k}}$ is continuous. The measure $P$ induces on $K_{k}$ a Radon measure $P_{k}$ and $\varphi_{k}\left(P_{k}\right)$ is a Radon measure on $L^{2}\left(0, T ; H^{-1}(G)\right) \times L^{2}\left(0, T ; H_{0}^{1}(G)\right)$. Since $\sigma$ is $\varphi_{k}\left(P_{k}\right)$ measurable, $\sigma \circ \varphi_{k}$ is $P$-measurable of $K_{k}$ in $L^{2}\left(0, T ; H^{-1}(G)\right) \times L^{2}\left(0, T ; H_{0}^{1}(G)\right)$. Let

$$
\left(z_{*, k}(\cdot, \omega), \hat{v}_{k}(\cdot, \omega)\right)= \begin{cases}\left(z_{*}(\cdot), \hat{v}(\cdot)\right)=(\sigma \circ \varphi)(\omega) & \text { if } \omega \in K_{k} \\ 0 & \text { if } \omega \notin K_{k}\end{cases}
$$

The functions $\left(z_{*, k}, \hat{v}_{k}\right)$ from $\Omega$ to $L^{2}\left(0, T ; H_{0}^{1}(G)\right) \times L^{2}\left(0, T ; H^{-1}(G)\right)$ are measurable and

$$
\left(z_{*, k}(\cdot, \omega), \hat{v}_{k}(\cdot, \omega)\right) \longrightarrow\left(z_{*}(\cdot, \omega), \hat{v}(\cdot, \omega)\right) \quad \text { a.s. }
$$

as $P\left(\bigcup_{k} K_{k}\right)=1$. Therefore $\left(z_{*}, \hat{v}\right)$ is $P$-measurable and $\sigma \circ \varphi$ is a measurable section of $\Lambda$.

We have $\left(z_{*}(\omega), \hat{v}(\cdot, \omega)\right) \in \Gamma(\alpha, \gamma(\cdot, \omega))$ and the lemma is proved. 
Proof of Theorem 2.1. It follows from (2.6) and from Lemmas 2.3-2.5 that

$$
\begin{aligned}
E\left(\|\Delta \bar{z}\|_{L^{2}\left(0, T ; H^{-1}(G)\right)}^{2}\right) & \leq C\left\{E\left(\|\tilde{v}\|_{L^{\infty}\left(0, T ; L^{2}(G)\right)}^{2}\right)+E\left(\left\|\bar{z}^{\prime}\right\|_{L^{2}\left(0, T ; H^{-1}(G)\right)}^{2}\right)\right\} \\
& \leq C\left\{\|\alpha\|_{L^{2}(G)}^{2}+E\left(\|\gamma\|_{L^{2}(G)}^{2}\right)\right\} .
\end{aligned}
$$

Set $v=-\tilde{v}-2 \Delta \bar{z}$, and the theorem is proved.

\section{Exact controllability: the linear case}

In this section, we will consider the problem

$$
\begin{gathered}
d y-\Delta y d t=-f d w+v d t \quad \text { in } G \times(0, T), \text { a.s., } \\
y(x, t ; \omega)=0 \quad \text { on } \partial G \times(0, T), \text { a.s., } \\
y(x, 0 ; \omega)=\alpha ; \quad y(x, T ; \omega)=\beta \quad \text { in } G, \text { a.s. }
\end{gathered}
$$

The main result of the section is the following theorem.

Theorem 3.1. Let $f$ be in $L^{2}\left(0, T ; L^{2}(G)\right)$ and let $\{\alpha, \beta\}$ be in $L^{2}(G)$. Then there exists a solution $\{v, y\}$ of (3.1), in

$$
L^{2}\left(\Omega, \mathcal{A}, P ; L^{2}\left(0, T ; H^{-1}(G)\right)\right) \times L^{2}\left(\Omega, \mathscr{A}, P ; L^{2}\left(0, T ; H_{0}^{1}(G)\right) \cap L^{\infty}\left(0, T ; L^{2}(G)\right)\right)
$$

and $y \in C\left(0, T ;\left(L^{2}(G)\right)_{\text {weak }}\right)$. Moreover

$$
\begin{aligned}
& E\left(\|y(\cdot, \omega)\|_{L^{\infty}\left(0, T ; L^{2}(G)\right)}^{2}\right)+E\left(\|y(\cdot, \omega)\|_{L^{2}\left(0, T ; H_{0}^{1}(G)\right)}^{2}\right)+E\left(\|v(\cdot ; \omega)\|_{L^{2}\left(0, T ; H^{-1}(G)\right)}^{2}\right) \\
& \leq C\left\{\mathscr{E}^{2}(\alpha, \beta)+E\left(\|f\|_{L^{2}\left(0, T ; L^{2}(G)\right)}^{2}\right)\right\}
\end{aligned}
$$

with

$$
\mathscr{E}(\alpha, \beta)=\|\alpha\|_{L^{2}(G)}+\|\beta\|_{L^{2}(G)} .
$$

The constant $C$ is independent of $\alpha, \beta, f, g$.

First we will consider the linear stochastic problem

$$
\begin{gathered}
d \hat{y}-\Delta \hat{y} d t=-f d w \quad \text { in } G \times(0, T), \\
\hat{y}(x, t ; \omega)=0 \quad \text { on } \partial G \times(0, T), \text { a.s. } \\
\hat{y}(x, 0 ; \omega)=0 \quad \text { in } G, \text { a.s. }
\end{gathered}
$$

It is well known that for a given $f \in L^{2}\left(\Omega, \mathcal{A}, P ; L^{2}\left(0, T ; L^{2}(G)\right)\right)$, there exists a unique solution $\hat{y}$ of (3.5) in $L^{2}\left(\Omega, \mathscr{A}, P ; L^{2}\left(0, T ; H_{0}^{1}(G)\right) \cap L^{\infty}\left(0, T ; L^{2}(G)\right)\right)$ with $\hat{y} \in C(0, T$; $\left.H^{-1}(G)\right)$ a.s. The discretisation of (3.5) leads to the following lemma. The result is known.

Lemma 3.2. Suppose all the hypotheses of Theorem 3.1 are satisfied. Then there exists

$$
\hat{y} \in L^{2}\left(\Omega, \mathscr{A}, P ; H_{0}^{1}(G)\right) \cap L^{\infty}\left(0, T ; L^{2}(G)\right)
$$


with $\hat{y} \in C\left(0, T ;\left(L^{2}(G)\right)_{\text {weak }}\right)$ a.s. such that

$$
\begin{gathered}
d \hat{y}-\Delta \hat{y} d t=f d w \quad \text { in } G, \text { a.s. } \\
\hat{y}(x, t ; \omega)=0 \quad \text { on } \partial G, \text { a.s. } \\
\hat{y}(x, 0 ; \omega)=0 \quad \text { in } G .
\end{gathered}
$$

Moreover

$$
E\left(\|\hat{y}\|_{L^{\infty}\left(0, T ; L^{2}(G)\right)}^{2}\right)+E\left(\|\hat{y}\|_{L^{2}\left(0, T ; H_{0}^{1}(G)\right)}^{2}\right) \leq C E\left(\|f\|_{L^{2}\left(0, T ; L^{2}(G)\right)}^{2}\right) .
$$

The constant $C$ is independent of $f$.

Proof of Theorem 3.1. Let $\hat{y}$ be as in Lemma 3.2. Since

$$
\hat{y}(\cdot, t)-\int_{0}^{t} \Delta \hat{y}(\cdot, s) d s=-\int_{0}^{t} f d w(s) \quad \text { a.s. } \forall t \in[0, T]
$$

we have $\hat{y} \in C\left(0, T ; H^{-1}(G)\right)$ a.s. and hence also since it is bounded in $L^{2}(G)$,

$$
\hat{y}(\cdot) \in C\left(0, T ;\left(L^{2}(G)\right)_{\text {weak }}\right) \text { a.s. }
$$

Therefore $\hat{y}(\cdot, T ; \omega) \in L^{2}(G)$ a.s. We set

$$
\gamma=\beta-\hat{y}(\cdot, T ; \omega)
$$

and we deduce from Theorem 2.1 that there exists a unique solution $\hat{z}$ of (2.3). Set

$$
y(x, t ; \omega)=\hat{y}(x, t ; \omega)+z(x, t ; \omega) .
$$

Then it is clear that $\{v, y\}$ is a solution of (3.1) and

$$
y(x, 0 ; \omega)=\hat{y}(x, 0 ; \omega)+z(x, 0 ; \omega)=\alpha ; \quad y(x, T ; \omega)=\hat{y}(x, T ; \omega)+\gamma=\beta .
$$

The stated estimate is an immediate consequence of those of Theorem 2.1 and of Lemma 3.2.

\section{The nonlinear case}

We will make the following assumption on the nonlinear mapping $g$.

Assumption 4.1. Let $g$ be a continuous mapping of $L^{2}\left(0, T ; L^{2}(G)\right)$ into $L^{2}\left(0, T ; L^{2}(G)\right)$. We assume that

$$
\|g(y)\|_{L^{2}\left(0, T ; L^{2}(G)\right)} \leq C\|y\|_{L^{2}\left(0, T ; L^{2}(G)\right)} \quad \forall y \in L^{2}\left(0, T ; L^{2}(G)\right) .
$$

The main result of the section is the following theorem. 
Theorem 4.2. Let $\{\alpha, \beta, f\}$ be in $\left(L^{2}(G)\right)^{2} \times L^{2}\left(0, T ; L^{2}(G)\right)$ and let $g$ be as in Assumption 4.1. Then there exists $\{v, y\}$ in

$$
L^{2}\left(\Omega, \mathcal{A}, P ; L^{2}\left(0, T ; H^{-1}(G)\right)\right) \times L^{2}\left(\Omega, \mathscr{A}, P ; L^{2}\left(0, T ; H_{0}^{1}(G)\right) \cap L^{\infty}\left(0, T ; L^{2}(G)\right)\right)
$$

with $y \in C\left(0, T ; H^{-1}(G)\right)$ a.s., solution of the stochastic equation

$$
\begin{gathered}
d y-\Delta y d t+f d w=-v d t+g(y) d t, \\
y(\cdot, 0 ; \omega)=\alpha ; \quad y(\cdot, T ; \omega)=\beta \quad \text { in } G, \text { a.s. }
\end{gathered}
$$

Let $\tilde{y} \in L^{2}\left(0, T ; L^{2}(G)\right)$ and consider the exact controllability of the linear heat equation

$$
\begin{gathered}
z^{\prime}-\Delta z=\hat{v}+g(\tilde{y}+\bar{z}) \quad \text { in } G \times(0, T), \\
z(x, t ; \omega)=0 \quad \text { on } \partial G \times(0, T), \\
z(x, 0)=0 ; \quad z(x, T)=0 \quad \text { in } G .
\end{gathered}
$$

Let $\lambda>0$ and set

$$
\widehat{z}(x, t)=\exp (-\lambda t) z, \quad z_{1}=e^{-\lambda t} \bar{z}
$$

then we get

$$
\begin{gathered}
\hat{z}^{\prime}+\lambda \hat{z}-\Delta \hat{z}=e^{-\lambda t} \hat{v}+e^{-\lambda t} g\left(e^{\lambda t} z_{1}+\tilde{y}\right) \quad \text { in } G \times(0, T), \\
\hat{z}=0 \quad \text { on } \partial G \times(0, T), \\
\hat{z}(x, 0)=0 ; \quad \hat{z}(x, T)=0 \quad \text { in } G .
\end{gathered}
$$

Let

$$
\begin{aligned}
\mathscr{B}= & \left\{z:\|z\|_{L^{2}\left(0, T ; L^{2}(G)\right)}^{2} \leq C\left(\mathscr{E}^{2}(\alpha, \beta)+E\left(\|f\|_{L^{2}\left(0, T ; L^{2}(G)\right)}^{2}\right)\right),\right. \\
& \|z\|_{L^{2}\left(0, T ; H_{0}^{1}(G)\right)}^{2} \leq C^{2} / \mathcal{c}_{G}^{2}\left(\mathscr{E}^{2}(\alpha, \beta)+E\left(\|f\|_{L^{2}\left(0, T ; L^{2}(G)\right)}^{2}\right)\right), \\
& \left.\left\|\left(e^{\lambda t} z\right)^{\prime}\right\|_{L^{2}\left(0, T ; H^{-1}(G)\right)}^{2} \leq 2 C^{2} \exp (2 \lambda T)\left\{\mathscr{E}^{2}(\alpha, \beta)+E\left(\|f\|_{L^{2}\left(0, T ; L^{2}(G)\right)}^{2}\right)\right\}\right\} .
\end{aligned}
$$

It follows from Aubin's theorem that $\mathscr{B}$ is a compact convex subset of $L^{2}\left(0, T ; L^{2}(G)\right)$. We deduce from Theorem 2.1 that for a given $z_{1} \in \mathscr{B}$, there exists a solution $\left\{\hat{z}, e^{-\lambda t} \hat{v}\right\}$ of (4.6). Let

$$
\mathscr{L}\left(z_{1}\right)=\left\{\hat{z}:\left\{\hat{z}, e^{-\lambda t} \hat{v}\right\} \text { solution of }(4.6)\right\}
$$

Then $\mathscr{L}$ is a set-valued mapping of $\mathscr{B}$ into the subsets of $L^{2}\left(0, T ; L^{2}(G)\right)$. 
Lemma 4.3. Let $\tilde{y}$ be in $L^{2}\left(0, T ; L^{2}(G)\right)$ with

$$
\|\tilde{y}\|_{L^{2}\left(0, T ; L^{2}(G)\right)} \leq C\left\{\mathscr{E}(\alpha, \beta)+E\left(\|f\|_{L^{2}\left(0, T ; L^{2}(G)\right)}\right)\right\}
$$

and let $\mathscr{L}$ be as in (4.8), then for large positive $\lambda$, the mapping takes $\mathscr{B}$ into $\mathscr{B}$. The images of $\mathscr{L}$ are closed convex subsets of $L^{2}\left(0, T ; L^{2}(G)\right)$.

Proof. (1) We have

$$
\begin{aligned}
& (2 \lambda-1)\|\hat{z}\|_{L^{2}\left(0, T ; L^{2}(G)\right)}^{2}+c_{G}^{2}\|\hat{z}\|_{L^{2}\left(0, T ; H_{0}^{1}(G)\right)}^{2} \\
& \quad \leq C\left\|e^{-\lambda t} g\left(\tilde{y}+e^{\lambda t} z_{1}\right)\right\|_{L^{2}\left(0, T ; L^{2}(G)\right)}+\left\|e^{-\lambda t} \hat{v}\right\|_{L^{2}\left(0, T ; H^{-1}(G)\right)} .
\end{aligned}
$$

With $z_{1} \in \mathscr{B}$, it follows from the estimates of Theorem 3.1 and from Assumption 4.1 that

$$
(2 \lambda-1)\|\hat{z}\|_{L^{2}\left(0, T ; L^{2}(G)\right)}^{2}+c_{G}^{2}\|\hat{z}\|_{L^{2}\left(0, T ; H_{0}^{1}(G)\right)}^{2} \leq C_{2}\left\{\mathscr{E}^{2}(\alpha, \beta)+E\left(\|f\|_{L^{2}\left(0, T ; L^{2}(G)\right)}^{2}\right)\right\} .
$$

We will take $\lambda$ such that $2 C_{2} /(2 \lambda-1) \leq C^{2}$. Thus,

$$
\|\widehat{z}\|_{L^{2}\left(0, T ; L^{2}(G)\right)}^{2} \leq C\left\{\mathscr{E}^{2}(\alpha, \beta)+E\left(\|f\|_{L^{2}\left(0, T ; L^{2}(G)\right)}^{2}\right)\right\} .
$$

It now follows that

$$
c_{G}^{2}\|\hat{z}\|_{L^{2}\left(0, T ; H_{0}^{1}(G)\right)}^{2} \leq C\left\{\mathscr{E}^{2}(\alpha, \beta)+E\left(\|f\|_{L^{2}\left(0, T ; L^{2}(G)\right)}^{2}\right)\right\} .
$$

We have

$$
\begin{aligned}
\left\|\left(e^{\lambda t} \hat{z}\right)^{\prime}\right\|_{L^{2}\left(0, T ; H^{-1}(G)\right)}^{2} & =\left\|\bar{z}^{\prime}\right\|_{L^{2}\left(0, T ; H^{-1}(G)\right)}^{2} \\
& \leq C\left\{(1+2 C \exp (\lambda T))\left\{\mathscr{E}^{2}(\alpha, \beta)+E\left(\|f\|_{L^{2}\left(0, T ; L^{2}(G)\right)}^{2}\right)\right\} .\right.
\end{aligned}
$$

It follows that $\mathscr{L}$ takes $\mathscr{B}$ into $\mathscr{B}$.

(2) We now show that the images of $\mathscr{L}$ are closed convex subsets of $L^{2}\left(0, T ; L^{2}(G)\right)$. Suppose that $\hat{z}_{1}, \hat{z}_{2}$ are in $\mathscr{L}\left(z_{1}\right)$ and set

$$
\widehat{z}=\mu \hat{z}_{1}+(1-\mu) \hat{z}_{2}, \quad \mu \in[0,1] .
$$

Then

$$
\begin{gathered}
\hat{z}^{\prime}-\Delta \hat{z}+\lambda \hat{z}=e^{-\lambda t}\left\{\mu \hat{v}_{1}+(1-\mu) \hat{v}_{2}\right\}+e^{-\lambda t} g\left(\tilde{y}+e^{\lambda t} z_{1}\right), \\
\hat{z}(x, t)=0 \quad \text { on } \partial G \times(0, T), \\
\hat{z}(x, 0)=0 ; \quad \hat{z}(x, T)=0 \quad \text { in } G .
\end{gathered}
$$

It is now clear that $\hat{z} \in \mathscr{L}\left(z_{1}\right)$. We now show that the images of $\mathscr{L}\left(z_{1}\right)$ are closed subsets of $L^{2}\left(0, T ; L^{2}(G)\right)$. Suppose that

$$
\widehat{z}_{n} \longrightarrow \widehat{z} \quad \text { in } L^{2}\left(0, T ; L^{2}(G)\right) ; \hat{z}_{n} \in \mathscr{L}\left(z_{1}\right) .
$$


Let $\left\{\hat{z}_{n}, e^{-\lambda t} v_{n}\right\}$ be a solution of the exact controllability problem

$$
\begin{aligned}
& \widehat{z}_{n}^{\prime}+\lambda \widehat{z}_{n}-\Delta \widehat{z}_{n}=e^{-\lambda t}\left\{g\left(\tilde{y}+z_{1}\right)+v_{n}\right\} \quad \text { in } G \times(0, T), \\
& \widehat{z}_{n}=0 \quad \text { on } \partial G \times(0, T), \\
& \hat{z}_{n}(x, 0)=0 ; \quad \widehat{z}_{n}(x, T)=0 \quad \text { in } G .
\end{aligned}
$$

From the estimates of Theorems 2.1 and 3.1, we get

$$
\left\|\hat{z}_{n}\right\|_{L^{2}\left(0, T ; H_{0}^{1}(G)\right)}+\left\|\hat{z}_{n}^{\prime}\right\|_{L^{2}\left(0, T ; H^{-1}(G)\right)}+\left\|v_{n}\right\|_{L^{T} 2\left(0, T ; H^{-1}(G)\right)} \leq C .
$$

It follows that there exists a subsequence such that

$$
\left\{\widehat{z}_{n_{j}}, \hat{z}_{n_{j}}^{\prime}, v_{n_{j}}\right\} \longrightarrow\left\{\hat{z}, \hat{z}^{\prime}, v\right\}
$$

in

$$
C\left(0, T ; L^{2}(G)\right) \cap\left(L^{2}\left(0, T ; H_{0}^{1}(G)\right)\right)_{\text {weak }} \times\left(L^{2}\left(0, T ; H^{-1}(G)\right)\right)_{\text {weak }}^{2}
$$

Since the problem is linear, it is clear that $\hat{z} \in \mathscr{L}\left(z_{1}\right)$ and the lemma is proved.

Lemma 4.4. The set valued mapping $\mathscr{L}$ mapping $\mathscr{B}$ into the closed convex subsets of $L^{2}(0, T$; $\left.L^{2}(G)\right)$, has a fixed point.

Proof. In view of Lemma 4.3, to apply the Kakutani fixed point theorem to the set valued mapping $\mathscr{L}$ we have to show that the mapping is u.s.c. and therefore it is sufficient to prove that its graph is closed. Suppose that

$$
\hat{z}_{n} \in \mathscr{L}\left(z_{1}^{n}\right) \quad \text { with }\left\{\hat{z}_{n}, z_{1}^{n}\right\} \longrightarrow\left\{\widehat{z}, z_{1}\right\} \quad \text { in }\left(L^{2}\left(0, T ; L^{2}(G)\right)\right)^{2} .
$$

We now show that $\hat{z} \in \mathscr{L}\left(z_{1}\right)$. With $\hat{z}_{n} \in \mathscr{L}\left(z_{1}^{n}\right)$, there exists $\hat{v}_{n} \in L^{2}\left(0, T ; H^{-1}(G)\right)$ such that

$$
\left\{\widehat{z}_{n}, e^{-\lambda t} \hat{v}_{n}\right\} \text { is a solution of (4.6). }
$$

From the estimates of Theorems 2.1 and 3.1, we get

$$
\left\{\widehat{z}_{n}, \widehat{z}_{n}^{\prime}, \widehat{v}_{n}\right\} \longrightarrow\left\{\hat{z}, \hat{z}^{\prime}, \hat{v}\right\}
$$

in

$$
C\left(0, T ; L^{2}(G)\right) \cap\left(L^{2}\left(0, T ; H_{0}^{1}(G)\right)\right)_{\text {weak }} \times\left(L^{2}\left(0, T ; H^{-1}(G)\right)\right)_{\text {weak }}^{2} .
$$

With Assumption 4.1, we have

$$
g\left(e^{\lambda t} \widehat{z}_{n}+\tilde{y}\right) \longrightarrow g\left(e^{\lambda t} \hat{x}+\tilde{y}\right) \quad \text { in }\left(L^{2}\left(0, T ; L^{2}(G)\right)\right)_{\text {weak }} .
$$

It is now clear that $\hat{z} \in \mathscr{L}\left(z_{1}\right)$. It now follows from the Kakutani fixed point theorem that there exists $\hat{z} \in \mathscr{B}$ such that $\hat{z} \in \mathscr{L}(\hat{z})$. The lemma is proved. 
Lemma 4.5. Let $\tilde{y}$ be as in Lemma 4.3, then there exists $\{z, \hat{v}\}$ in

$$
L^{2}\left(0, T ; H_{0}^{1}(G)\right) \cap C\left(0, T ; L^{2}(G)\right) \times L^{2}\left(0, T ; H^{-1}(G)\right),
$$

such that

$$
\begin{gathered}
z^{\prime}-\Delta z=\hat{v}+g(\tilde{y}+z), \\
z(x, t)=0 \quad \text { on } \partial G \times(0, T), \\
z(x, 0)=0 ; \quad z(x, T)=0 \quad \text { in } G .
\end{gathered}
$$

Moreover

$$
\|z\|_{L^{2}\left(0, T ; H_{0}^{1}(G)\right)}+\left\|z^{\prime}\right\|_{L^{2}\left(0, T ; H^{-1}(G)\right)}+\|\hat{v}\|_{L^{2}\left(0, T ; H^{-1}(G)\right)} \leq C^{\mathscr{E}}(\alpha, \beta) .
$$

Furthermore the set valued mapping

$$
\Gamma(\tilde{y})=\{z:\{z, \hat{v}\} \text { solution of }(4.28)\}
$$

of $L^{2}\left(0, T ; L^{2}(G)\right)$ into $L^{2}\left(0, T ; H_{0}^{1}(G)\right)$, has non empty closed images and its graph is closed. Proof. (1) The existence of a solution of (4.28) is an immediate consequence of Lemma 4.4. We now show that the images of $\Gamma$ are closed. Suppose that

$$
z_{n} \in \Gamma(\tilde{y}) \quad \text { with } z_{n} \longrightarrow z \text { in } L^{2}\left(0, T ; H_{0}^{1}(G)\right) .
$$

With the estimates of the theorem, we have

$$
\left\|z_{n}^{\prime}\right\|_{L^{2}\left(0, T ; H^{-1}(G)\right)}+\left\|\hat{v}_{n}\right\|_{L^{2}\left(0, T ; H^{-1}(G)\right)} \leq C \text {. }
$$

Thus there exists a subsequence such that

$$
\left\{z_{n_{j}}, z_{n_{j}}^{\prime}, \hat{v}_{n_{j}}\right\} \longrightarrow\left\{z, z^{\prime}, \hat{v}\right\}
$$

in

$$
L^{2}\left(0, T ; H_{0}^{1}(G)\right) \cap C\left(0, T ; L^{2}(G)\right) \times\left(L^{2}\left(0, T ; H^{-1}(G)\right)\right)_{\text {weak }}^{2}
$$

With Assumption 4.1 on $g$, it is easy to check that indeed $z \in \Gamma(\tilde{y})$.

(2) We now show that the graph of $\Gamma$ is closed. Suppose that

$$
\left\{z_{n}, \tilde{y}_{n}\right\} \longrightarrow\{z, \tilde{y}\} \quad \text { in } L^{2}\left(0, T ; H^{1}(G)\right) \times L^{2}\left(0, T ; L^{2}(G)\right) \text { with } z_{n} \in \Gamma\left(\tilde{y}_{n}\right) .
$$

Then from the estimates of the theorem, we get as before

$$
\left\{z_{n_{j}}, z_{n_{j}}^{\prime}, \hat{v}_{n_{j}}\right\} \longrightarrow\left\{z, z^{\prime}, \hat{v}\right\}
$$

in

$$
L^{2}\left(0, T ; H_{0}^{1}(G)\right) \cap C\left(0, T ; L^{2}(G)\right) \times\left(L^{2}\left(0, T ; H^{-1}(G)\right)\right)_{\text {weak }}^{2}
$$


With Assumption 4.1 on $g$, we obtain

$$
g\left(\tilde{y}_{n_{j}}+z_{n_{j}}\right) \longrightarrow g(\tilde{y}+z) \quad \text { in }\left(L^{2}\left(0, T ; L^{2}(G)\right)\right)_{\text {weak }} .
$$

It is now clear that $z \in \Gamma(\tilde{y})$. The lemma is proved.

We now turn to the case when $\tilde{y}$ is in $L^{2}\left(\Omega, \mathscr{A}, P ; L^{2}\left(0, T ; L^{2}(G)\right)\right)$.

Theorem 4.6. Suppose all the hypotheses of Theorem 4.2 are satisfied and let $\tilde{y}$ be as in Theorem 3.1. Then there exists $\{\tilde{v}, \tilde{z}\}$, solution of

$$
\begin{gathered}
\tilde{z}^{\prime}-\Delta \tilde{z}=\tilde{v}+g(\tilde{y}+\tilde{z}) \quad \text { in } G \times(0, T) \text { a.s. } \\
\tilde{z}(x, t ; \omega)=0 \quad \text { on } \partial G \times(0, T) \text { a.s. } \\
\tilde{z}(x, 0 ; \omega)=0 ; \quad \tilde{z}(x, T ; \omega)=0 \quad \text { in } G \text { a.s. }
\end{gathered}
$$

Moreover

$$
E\left(\|\tilde{z}\|_{L^{2}\left(0, T ; H_{0}^{1}(G)\right)}^{2}\right)+E\left(\|\tilde{z}\|_{L^{\infty}\left(0, T ; L^{2}(G)\right)}^{2}\right)+E\left(\|\widetilde{v}\|_{L^{2}\left(0, T ; H^{-1}(G)\right)}^{2}\right) \leq C_{\mathscr{E}}^{\mathscr{C}}(\alpha, \beta) .
$$

The constant $C$ is independent of $\tilde{y}, \alpha, \beta$.

Proof. It follows from Lemma 4.5 and from a theorem of Von Neumann that there exists a universally measurable section $\sigma$ of the set valued mapping $\Gamma$.

(1) Let $\{z, \hat{v}\}$ be as in Lemma 4.5 and let $\varphi$ be the random variable

$$
\varphi(\omega)=\tilde{y}(\cdot, \omega)
$$

of $\Omega \rightarrow L^{2}\left(0, T ; L^{2}(G)\right)$. Let

$$
\left.G \omega=\{\widetilde{z}(\cdot, \omega), \widetilde{v}(\cdot, \omega)\} \in L^{2}\left(0, T ; H_{0}^{1}(G)\right) \times L^{2}\left(0, T ; H^{-1}(G)\right): \text { solution of }(4.28)\right\}
$$

with $\tilde{y}$ replaced by $\tilde{y}(\cdot ; \omega)$. Thus, $G=\Gamma \circ \varphi$.

Since $P$ is a Radon measure and $\varphi$ is a random variable, there exists a compact subset $K_{k}$ of $\Omega$ such that

$$
P\left(\Omega / K_{k}\right) \leq 1 / k
$$

We may assume without loss of generality that the sets $K_{k}$ are increasing and that $P\left(\bigcup K_{n}\right)=1$.

We now show that $\sigma \circ \varphi$ is a measurable section of the set valued mapping $G$.

(2) Let $\varphi_{k}=\left.\varphi\right|_{K_{k}}$, then $P$ induces on $K_{k}$ a Radon measure $P_{k}$ and $\varphi_{k}\left(P_{k}\right)$ is a Radon measure on $L^{2}\left(0, T ; L^{2}(G)\right)$. Since $\sigma$ is $\varphi_{k}\left(P_{k}\right)$ measurable, $\sigma \circ \varphi_{k}$ is $P$-measurable from $K_{k}$ to $L^{2}\left(0, T ; H_{0}^{1}(G)\right) \times L^{2}\left(0, T ; H^{-1}(G)\right)$. Set

$$
\left\{\widetilde{z}_{k}(\cdot, \omega), \widetilde{v}_{k}(\cdot, \omega)\right\}= \begin{cases}\{\widetilde{z}(\cdot, \omega), \widetilde{v}(\cdot, \omega)\}=(\sigma \circ \varphi)(\omega) & \text { if } \omega \in K_{k}, \\ 0 & \text { if } \omega \notin K_{k} .\end{cases}
$$


Then the functions $\left\{\tilde{z}_{k}, \tilde{v}_{k}\right\}$ from $\Omega$ into $L^{2}\left(0, T ; H_{0}^{1}(G)\right) \times L^{2}\left(0, T ; H^{-1}(G)\right)$ are measurable. Moreover

$$
\left\{\widetilde{z}_{k}(\cdot, \omega), \widetilde{v}_{k}(\cdot, \omega)\right\} \longrightarrow\{\tilde{z}(\cdot, \omega), \widetilde{v}(\cdot, \omega)\}
$$

a.s. since $P\left(\bigcup_{k} K_{k}\right)=1$. Therefore $\sigma \circ \varphi$ is a measurable section of $G$. The theorem is proved.

Proof of Theorem 4.2. Let $\{\tilde{y}, v\}$ be as in Theorem 3.1 and let $\{\tilde{z}, \tilde{v}\}$ be as in Theorem 4.6. Set $\{y, \hat{v}\}=\{\tilde{y}+\widetilde{z}, v+\widetilde{v}\}$ and the theorem is proved.

\section{References}

[1] B. A. Ton, Exact controllability for a semilinear wave equation with both interior and boundary controls, to appear in Abstract and Applied Analysis, 2005.

[2] A. Bensoussan and R. Temam, Équations stochastiques du type Navier-Stokes, Journal of Functional Analysis 13 (1973), no. 2, 195-222 (French).

[3] C. Fabre, J.-P. Puel, and E. Zuazua, Contrôlabilité approchée de l'équation de la chaleur semilinéaire [Approximate controllability for the semilinear heat equation], Comptes Rendus de l'Académie des Sciences. Série I. Mathématique 315 (1992), no. 7, 807-812 (French).

[4] G. Lebeau and L. Robbiano, Contrôle exact de l'équation de la chaleur [Exact control of the heat equation], Communications in Partial Differential Equations 20 (1995), no. 1-2, 335-356 (French).

[5] D. L. Russell, A unified boundary controllability theory for hyperbolic and parabolic partial differential equations, SIAM Studies in Applied Mathematics 52 (1973), no. 3, 189-211.

[6] E. Zuazua, Exact boundary controllability for the semilinear wave equation, Nonlinear Partial Differential Equations and Their Applications. Collège de France Seminar, Vol. X (Paris, 19871988), Pitman Res. Notes Math. Ser., vol. 220, Longman Science Technology, Harlow, 1991, pp. $357-391$.

Bui An Ton: Department of Mathematics, University of British Columbia, Vancouver, BC, Canada V6T $1 Z 2$

E-mail address: bui@math.ubc.ca 


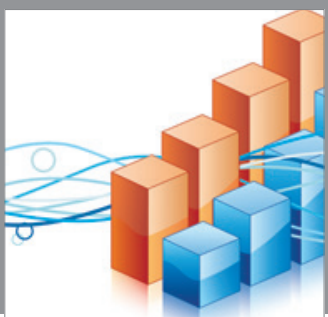

Advances in

Operations Research

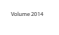

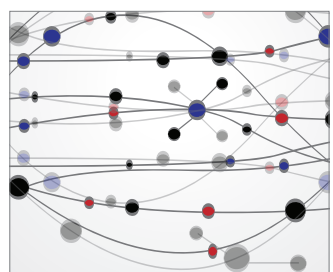

\section{The Scientific} World Journal
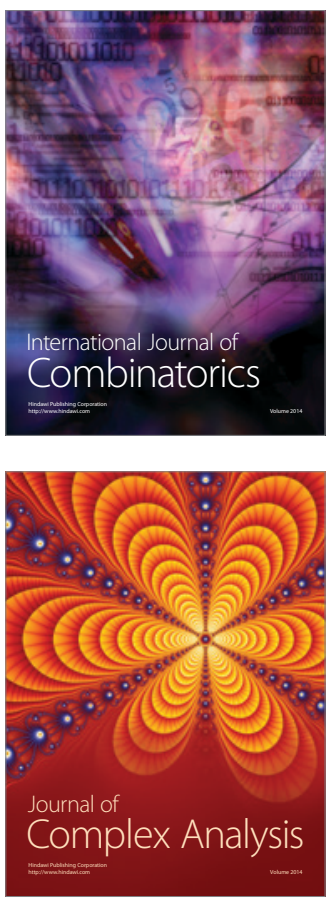

International Journal of

Mathematics and

Mathematical

Sciences
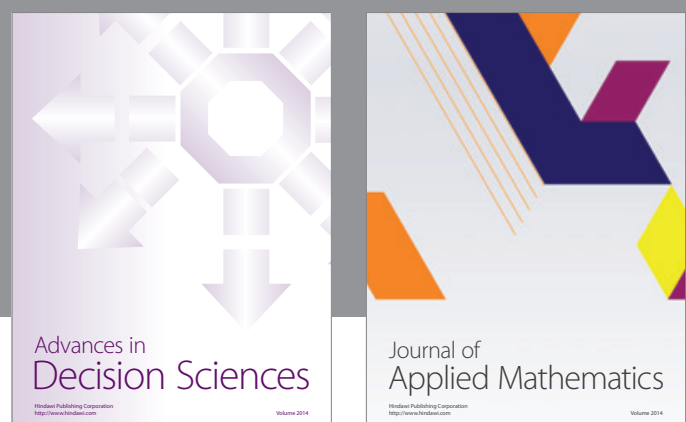

Journal of

Applied Mathematics
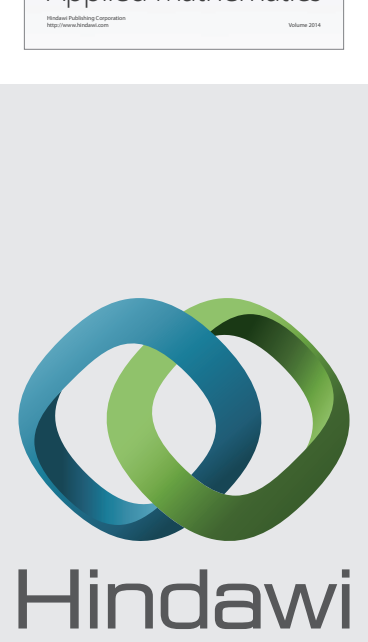

Submit your manuscripts at http://www.hindawi.com
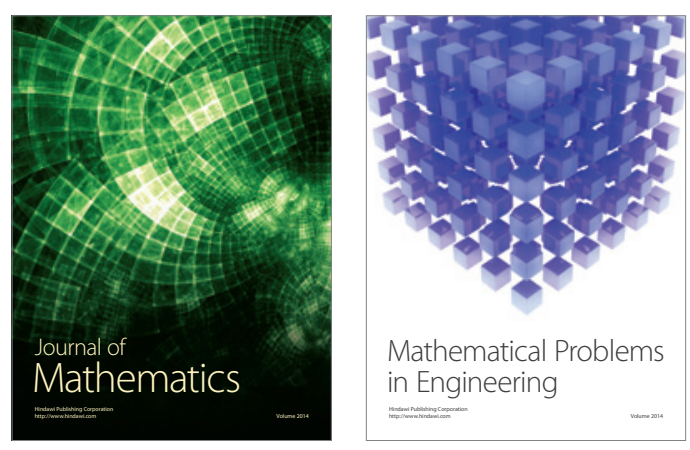

Mathematical Problems in Engineering
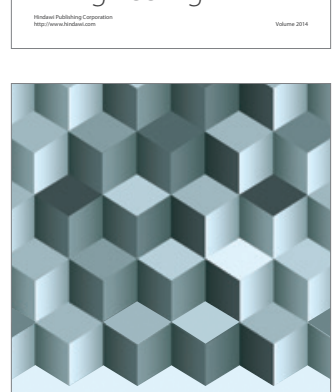

Journal of

Function Spaces
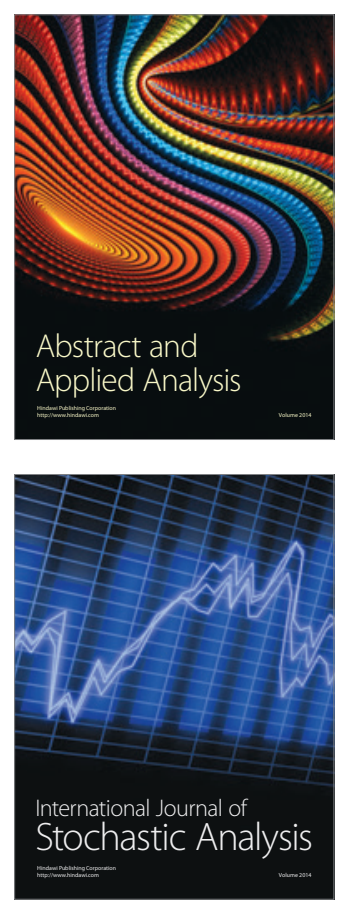

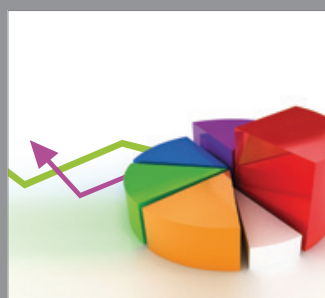

ournal of

Probability and Statistics

Promensencen
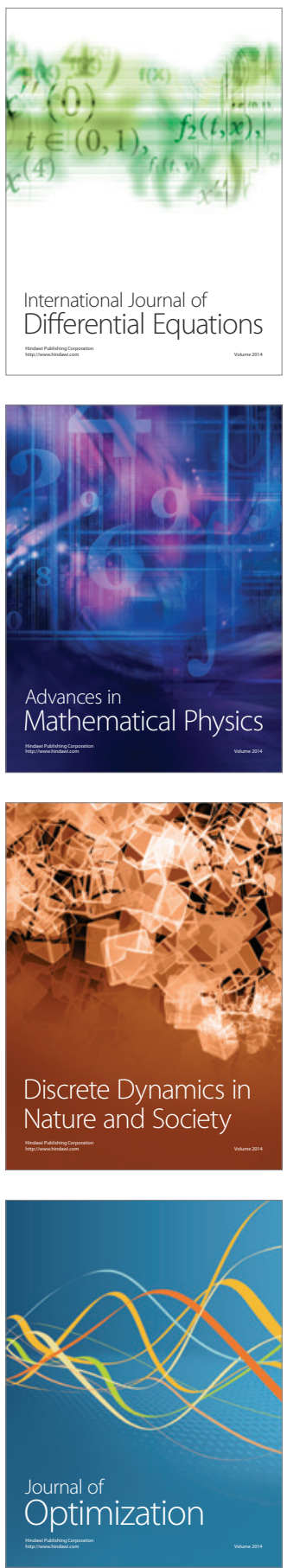